\title{
KNOTS WITHOUT UNKNOTTED INCOMPRESSIBLE SPANNING SURFACES
}

\author{
HERBERT C. LYON
}

\begin{abstract}
We construct a tame knot in $S^{3}$ which has no unknotted incompressible spanning surface.
\end{abstract}

I. Introduction. Schaufele [6] has exhibited tame knots in $S^{3}$ which have both knotted minimal spanning surfaces and unknotted incompressible spanning surfaces of higher genus. The Seifert construction [1, p. 140] yields an unknotted spanning surface for every knot. These observations led us to ask [3, p. 42] if every knot has an unknotted incompressible spanning surface. The answer is negative, with a counterexample provided by the knot in Figure 2, and the same techniques can be used to construct more counterexamples. Thus we establish the existence of a new class of knots; viz., those knots which have unknotted incompressible spanning surfaces. This new class lies properly between the Neuwirth knots and all knots. The $Z t$ module structure of the abelianized commutator subgroup of our knot is isomorphic to that of the square knot. Hence it is impossible to obtain an algebraic characterization of this new class of knots in terms of many of the classical tools of knot theory; e.g., Alexander matrices. Rice [5] has done some preliminary work with this class of knots.

The knot in Figure 2 also provides a counterexample to a conjecture of $[3$, p. 44]. All work is done in the $P L$ category, all surfaces are orientable and properly embedded, all knots are in $S^{3}$, and the terminology and notation follow that of [3] and [4].

II. The construction. Let $Q$ be the knot space of the nontrivial torus knot $(p, q)$, with longitude $\lambda$ and unique [2] meridian $\mu$.

LEMMA. If $B \subset Q$ is a connected, incompressible, and boundary incompressible bounded surface, then no component of $\mathrm{Bd} B$ is isotopic, in $\mathrm{Bd} Q$, to $\mu$.

Proof. If we assume the contrary, then $\mathrm{Bd} B$ lies in an annulus $A \subset \mathrm{Bd} Q$, and each component of $\mathrm{Bd} A$ is isotopic, in $\mathrm{Bd} Q$, to $\mu$. Let

Presented to the Society, April 21, 1971; received by the editors June 18, 1971 and, in revised form, February 7, 1972.

AMS 1970 subject classifications. Primary 55A25; Secondary 55A05, 55A35.

Key words and phrases. Genus, incompressible surface, knot, knot space, knotted surface, Neuwirth knot, spanning surface. 
$Q_{1}$ and $Q_{2}$ be two copies of $Q$, with subscripts identifying respective subspaces. If we join $Q_{1}$ and $Q_{2}$ by identifying $A_{1}$ with $A_{2}$ and $B_{1} \cap A_{1}$ with $B_{2} \cap A_{2}$, then the closed incompressible surface $B_{1} \cup B_{2}$ must separate the resulting knot space. Thus $B$ separates $Q$, so $\pi_{1}(Q)$ can be written as a nontrivial free product with amalgamation along $\pi_{1}(B)$. But $\pi_{1}(Q)$ has nontrivial center, so $\pi_{1}(B)=Z$; i.e., $B$ is an annulus. This contradicts the fact that the torus knot $(p, q)$ is prime.

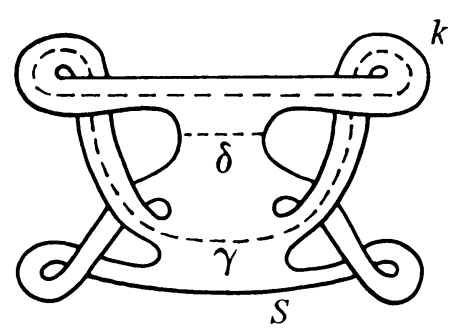

Figure 1

Consider the square knot $k$ and its spanning surface $S$ as shown in Figure 1. The manifold $\mathrm{Cl}\left(S^{3}-N(k)\right)$ is fibered over $S^{\mathbf{1}}$, with $S \cap$ $\mathrm{Cl}\left(S^{3}-N(k)\right)$ as fiber. Note that the curve $\gamma$ is chosen so that $N(\gamma) \cap S$ is an unknotted and untwisted annulus. Thus there is a properly embedded nonsingular disk $D \subset \mathrm{Cl}\left(S^{3}-N(\gamma)\right)$ with $(\mathrm{Bd} D) \cap S=\varnothing$. This disk $D$ may also be chosen so that $D \cap S=\delta \subset$ Int $D$, where $\delta \subset S$ is the spanning arc indicated in Figure 1. The surface $\mathrm{Bd} N(\gamma)$ is incompressible in $\mathrm{Cl}\left(S^{3}-(N(k) \cup N(\gamma))\right)$. Let $\alpha$ be the oriented boundary of a nonseparating disk in $N(\gamma)$, and let $\beta$ be the oriented curve $D \cap B d N(\gamma)$, so that $\alpha$ and $\beta$ form a basis for $\pi_{1}(\mathrm{Bd} N(\gamma))=Z \times Z$.

Remove Int $N(\gamma)$ from $S^{3}$ and replace it with $Q$ sewn in so that $\alpha$ is identified with $\lambda$ and $\beta$ with $\mu$. The disk $D$ is sewn along $\mu$, so the resulting manifold $V$ is still homeomorphic to $S^{3}$, and $\mathrm{Cl}(V-N(k))=M$ is a knot space. The corresponding knot, for the case $(p, q)=(2,3)$, is shown in Figure 2.

THEOREM 1. If $T$ is any incompressible spanning surface in the knot space $M$, then $T$ is knotted.

Proof. We prove this by moving $T$ with an isotopy until it misses $Q$. The surface $S$ separates $\mathrm{Bd} N(\gamma)=\mathrm{Bd} Q$ into two annuli, whose closures we denote by $A_{1}$ and $A_{2}$. Each component of $A_{1} \cap A_{2}$ is a meridian in Bd $Q$. If $S^{\prime}=(S \cap M) \cup A_{1}$, then $S^{\prime}$ is also a spanning surface in $M$. Put $T$ in general position with $S^{\prime}$ and assume the number of components in $S^{\prime} \cap T$ is minimal. 
If $S^{\prime} \cap T \neq \varnothing$, the geometric structure of the covering corresponding to the commutator subgroup of $\pi_{1}(M)$ assures us that there is some component of $T \cap\left(M-S^{\prime}\right)$ whose closure $T^{\prime}$ meets $S^{\prime}$ only from the side opposite $A_{2}$. Put $T^{\prime}$ in general position with $A_{2}$ and assume the number of components in $T^{\prime} \cap A_{2}$ is minimal. Now $M-\left(S^{\prime} \cup Q\right)$ was constructed so as to have a product structure, so if $T^{\prime} \cap A_{2}=\varnothing$, we know from Waldhausen [7] that $T^{\prime}$ is parallel to a surface in $S^{\prime}$. Hence we can move $T$ by an isotopy which reduces the number of components in $T \cap S^{\prime}$, a contradiction.

If $T^{\prime} \cap A_{2} \neq \varnothing$, then each component of $T^{\prime} \cap A_{2}$ is a meridian in $\mathrm{Bd} Q$. Put $T$ in general position with $\mathrm{Bd} Q$ without moving $T^{\prime} \cap A_{2}$. Our lemma assures us that each component of $T \cap Q$ is boundary parallel and hence may be removed by an isotopy. If $S^{\prime} \cap T=\varnothing$, then $T \cap A_{2}$ may be assumed to be either empty or to consist entirely of meridians in $\mathrm{Bd} Q$, and similar reasoning allows us to remove each component of $T \cap Q$.

COROLlaRY. Let $T_{i}, 1 \leqq i \leqq n$, be a set of pairwise disjoint incompressible spanning surfaces in $M$. Then some component of $\mathrm{Cl}\left(M-\bigcup_{i=1}^{n} N\left(T_{i}\right)\right)$ is not a cube-with-handles.

Thus we have a counterexample to our conjecture [3, p. 44] that every knot space $M$ contains a finite system $T_{i}, 1 \leqq i \leqq n$, of pairwise disjoint incompressible spanning surfaces such that:

(a) Each component of $\mathrm{Cl}\left(M-\bigcup_{i=1}^{n} N\left(T_{i}\right)\right)$ is a cube-with-handles, and

(b) any additional disjoint incompressible spanning surface is parallel to one of the $T_{i}$ 's.

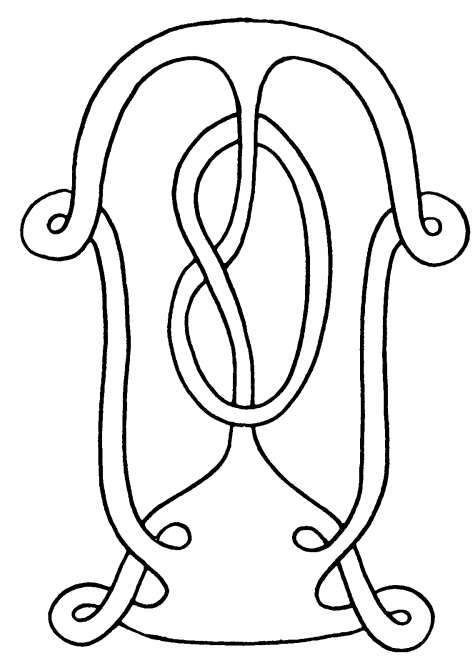

FIGURE 2 
Let $G_{1}=\pi_{1}(M)$, let $G_{2}$ be the group of the square knot, let $G_{i}^{\prime}=\left[G_{i}, G_{i}\right]$, and let $G_{i}^{\prime \prime}=\left[G_{i}^{\prime}, G_{i}^{\prime}\right]$ for $i=1,2$.

Theorem 2. The $Z t$ modules $G_{i}^{\prime} / G_{i}^{\prime \prime}$ are isomorphic, for $i=1,2$.

Proof. We saw in the proof of Theorem 1 that an incompressible spanning surface for $M$ may be assumed to miss $Q$. Hence, if $\varphi: \tilde{M} \rightarrow M$ denotes the covering of $M$ corresponding to $G_{1}^{\prime}$, then $\varphi$ is a homeomorphism when restricted to any component of $\varphi^{-1}(Q)$. But $M$ was constructed in such a way that if we remove $Q$ and sew in a solid torus $W^{\prime}$ so that $\alpha$ bounds a nonseparating disk in $W^{\prime}$, we will have the square knot space. The corresponding operation lifted to $\tilde{M}$ converts $\tilde{M}$ into the covering of the square knot space corresponding to $G_{2}^{\prime}$. In either case, the curves $\varphi^{-1}(\beta)$ will generate all of the homology contributed by either $\varphi^{-1}(Q)$ or $\varphi^{-1}\left(W^{\prime}\right)$, and the curves $\varphi^{-1}(\alpha)$ will all be homologous to zero.

\section{BIBLIOGRAPHY}

1. R. H. Fox, A quick trip through knot theory, Topology of 3-Manifolds and Related, Topics (Proc. Univ. of Georgia Inst., 1961), Prentice-Hall, Englewood Cliffs, N.J., 1962, pp. 120-167. MR 25 \#3522.

2. John Hempel, A simple connected 3-manifold is $S^{3}$ if it is the sum of a solid torus and the complement of a torus knot, Proc. Amer. Math. Soc. 15 (1964), 154-158. MR 28 \#599.

3. Herbert C. Lyon, Incompressible surfaces in knot spaces, Ph. D. Dissertation, University of Michigan, Ann Arbor, Mich., 1970.

4. - Incompressible surfaces in knot spaces, Trans. Amer. Math. Soc. 157 (1971), 53-62. MR 43 \#1169.

5. P. M. Rice, On knot groups, University of Georgia, Athens, Ga. (Preprint).

6. C. B. Schaufele, The commutator group of a doubled knot, Duke Math. J. 34 (1967), 677-681. MR 35 \#7328.

7. Friedhelm Waldhausen, On irreducible 3-manifolds which are sufficiently large, Ann. of Math. (2) 87 (1968), 56-88. MR 36 \#7146.

Department of Mathematics, University of Michigan-Flint, Flint, Michigan 48503

Department of Mathematics, University of Jordan, amman, The Hashemite KINGDOM OF JORDAN 